\title{
Lower Antiplatelet Effect of Aspirin in Essential Thrombocythemia than in Coronary Artery Disease
}

\author{
Oliver Buchhave Pedersen ${ }^{1,2}$ (1) \\ Anne-Mette Hvas ${ }^{1,3}$ \\ Erik Lerkevang Grove 2,3 (1)

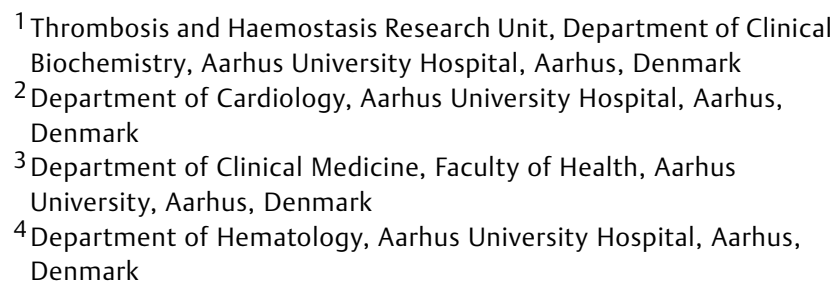

Hans Beier Ommen ${ }^{4}$

Steen Dalby Kristensen ${ }^{2,3}$

Address for correspondence Oliver Buchhave Pedersen, MD, Aarhus University Hospital, Palle Juul-Jensens Boulevard 99, 8200 Aarhus N, Denmark (e-mail: olihped@gmail.com).

TH Open 2021;5:e230-e238.

\section{Abstract \\ Keywords \\ - aspirin \\ - essential thrombocythemia \\ - platelet aggregation \\ - thromboxane B2 \\ - platelet activation \\ - platelet function test \\ - coronary artery disease}

Background Patients with essential thrombocythemia (ET) and coronary artery disease (CAD) have increased risk of thromboembolic complications. In addition, a reduced antiplatelet effect of aspirin has been demonstrated in both patient groups. As ET is a platelet disorder, platelets may be more important for the thromboembolic risk in $\mathrm{ET}$ than in CAD. We aimed to investigate the antiplatelet effect of aspirin and platelet turnover in ET versus CAD patients.

Methods We included $48 \mathrm{ET}$ patients and an age-matched group of 48 CAD patients. The effect of aspirin was evaluated by thromboxane $B_{2}\left(T X B_{2}\right)$ levels and platelet aggregation. Platelet turnover was assessed by immature platelet count (IPC) and immature platelet fraction (IPF).

Results ET patients had reduced effect of aspirin compared with CAD patients, demonstrated by significantly higher $\mathrm{TXB}_{2}$ levels (median of differences $=22.3 \mathrm{ng} / \mathrm{mL}$, $p<0.0001$ ) and platelet aggregation (median of differences $=131.0 \mathrm{AU}^{*} \mathrm{~min}$, $p=0.0003)$. Furthermore, ET patients had significantly higher IPC $(p<0.0001)$ and IPF $(p=0.0004)$ than CAD patients.

Conclusion ET patients have lower 24-hour antiplatelet effect of aspirin than CAD patients. This may be explained by an increased platelet production and turnover counteracting the antiplatelet effect of aspirin. These findings strengthen the rationale for exploring novel antiplatelet regimens in ET patients to reduce the risk of cardiovascular events. received

February 2, 2021

accepted after revision April 16, 2021
DOI https://doi.org/

10.1055/s-0041-1731309. ISSN 2512-9465.

\section{(C) 2021. The Author(s).}

This is an open access article published by Thieme under the terms of the Creative Commons Attribution License, permitting unrestricted use, distribution, and reproduction so long as the original work is properly cited. (https://creativecommons.org/licenses/by/4.0/)

Georg Thieme Verlag KG, Rüdigerstraße 14, 70469 Stuttgart, Germany 


\section{Introduction}

Patients diagnosed with essential thrombocythemia (ET) or coronary artery disease (CAD) both receive aspirin due to an increased thromboembolic risk. ${ }^{1,2}$ However, in both patient groups, a reduced effect of aspirin has been demonstrated. ${ }^{3-7}$ It is currently not clear whether these patients' groups have different antiplatelet effect of aspirin and if so, whether differences in platelet turnover might contribute to the underlying mechanisms.

ET is a myeloproliferative disorder characterized by increased platelet count and turnover primarily due to high platelet generation. ${ }^{8,9}$ The increased thromboembolic risk in ET cannot solely be explained by increased platelet count, since extreme thrombocythemia with counts higher than $1000 \times 10^{9} / \mathrm{L}$ has been associated with lower risk of thrombosis, ${ }^{4}$ perhaps explained by acquired von Willebrand's disease. ${ }^{10}$ Increased platelet turnover may be an important factor to explain the reduced effect of aspirin not only in ET patients but also in other patient groups. ${ }^{3,8}$

An increased platelet turnover results in an increased amount of reticulated, immature platelets in the circulation. ${ }^{11}$ Immature platelets counteract the irreversible inactivation of platelets by aspirin due to release of newly formed platelets unaffected by aspirin. ${ }^{11}$ Notably, the effect of aspirin has been found to be reduced particularly at the end of the usual 24-hour dosing interval. ${ }^{12-14}$ This has fostered an interest in exploring the effect of other dosing regimens of aspirin in ET patients. ${ }^{15,16}$ Additionally, immature platelets are larger and suggested to be more reactive than mature platelets, likely explained by residual ribonucleic acid (RNA) content that provides the ability to produce prothrombotic proteins contributing to increased platelet activation. ${ }^{11,17-20}$

We investigated ET patients matched with stable CAD patients to explore differences in aspirin response and differences in platelet turnover as a proof of that platelets may have different impact on thromboembolic risk in ET than in CAD. The differential effects of $75 \mathrm{mg}$ aspirin daily at 24 and 1 hour after aspirin intake were evaluated by thromboxane $\mathrm{B}_{2}\left(\mathrm{TXB}_{2}\right)$ and platelet aggregation measurements. We hypothesized that (1) ET patients have lower effect from aspirin during 24 hours than CAD patients, and (2) ET patients have higher platelet turnover demonstrated by higher immature platelet count (IPC) and immature platelet fraction (IPF) than CAD patients.

\section{Materials and Methods}

\section{Study Population and Design}

The study was an observational cohort study on 48 ET patients compared with data on 48 age-matched CAD patients obtained from a previous publication. ${ }^{12}$ ET patients were included at the Department of Hematology by the attending physicians. All ET patients were diagnosed based on the World Health Organization (WHO) criteria ${ }^{21}$ and all matched CAD patients had angiographically documented stable CAD. All included patients were older than 18 years and treated with nonenteric coated aspirin mono antiplatelet therapy ( $75 \mathrm{mg}$ once daily) for at least 1 month upon study enrolment. Patients were excluded if they received any antithrombotic therapy other than aspirin. The study was approved by The Central Denmark Region Committees in Biomedical Research Ethics (Reference number: 1-10-72426-17) and by the Danish Data Protection Agency (Journal number: 1-16-02-916-17). Informed consent was obtained from all patients, and the study was conducted in accordance with the Helsinki-II Declaration.

\section{Blood Sampling}

Patients had one blood sample obtained from an antecubital vein using a 21-gauge needle with minimum of stasis 1 hour after aspirin intake and another blood sample 24 hours after aspirin intake. Platelet count, red blood cell count, white blood cell count, platelet distribution width, mean platelet volume, IPC, and IPF were assessed in whole blood anticoagulated with ethylenediaminetetraacetic (Becton Dickinson Bioscience, California, United States) using an automated hematological analyzer (Sysmex XN-9000, Norderstedt, Germany). Plasma-creatinine was analyzed in lithium-heparin tubes (Becton Dickinson Bioscience) using Cobas 6000 (Roche, Basel, Switzerland).

\section{Thromboxane $B_{2}$}

Serum $\mathrm{TXB}_{2}$ was measured using an enzyme-linked immunosorbent assay according to manufacturer's instructions (Cayman Chemical, Ann Arbor, Michigan, United States). After exactly 1 hour of clotting at $37^{\circ} \mathrm{C}$, serum was collected after centrifugation in 10 minutes at $2,600 \mathrm{~g}$ and stored at $-80^{\circ} \mathrm{C}$ until analysis. All samples were measured in duplicate. Samples with results outside the standard curve were reanalyzed with appropriate dilutions. In addition, samples were reanalyzed if the variation in the duplicate measurements was above $20 \%$.

\section{Platelet Aggregation}

Hirudin tubes (Roche, Basel, Switzerland) were used to collect whole blood for multiple electrode aggregometry analysis of platelet aggregation. Samples rested for 30 minutes after collection and analyzed within 2 hours using the Multiplate Analyser (Roche, Basel, Switzerland). ${ }^{22}$ To induce platelet aggregation, arachidonic acid (ASPItest $0.5 \mathrm{mM}$ ) was used as agonist. Platelet aggregation was quantified as area under the curve (AUC, aggregation units [AU] $\mathrm{x}$ minutes). Measurements were repeated if AUC by each of the two electrodes pairs varied more than $20 \%$ from mean.

\section{Statistics and Sample Size Calculation}

The distribution of all data was evaluated by Q-Q plots and histograms. Continuous data were described as mean \pm standard deviation when data were normally distributed and, if not, as median and interquartile range (IQR). Categorical data were described by percentages. Differences between ET patients and CAD patients were analyzed with an unpaired t-test for normally distributed data and with MannWhitney U test for nonnormally distributed data. Correlation analyses were performed with Spearman's $\rho$ for data not 
following normal distribution. All tests of significance were two-tailed, with a probability value of $p<0.05$. All statistics was performed in GraphPad Prism 6 (GraphPad Software Inc., La Jolla, California, United States).

The primary outcome was the difference in delta values (value 1 hour after aspirin intake minus the value 24 hours after aspirin intake) of $\mathrm{TXB}_{2}$ between the two patient groups. Using our previously published data, the mean delta value in $\mathrm{TXB}_{2}$ levels in ET patients was found to be $23 \mathrm{ng} / \mathrm{mL}$. $^{23}$ From published data with CAD patients, the mean delta value in $\mathrm{TXB}_{2}$ levels was found to be $5 \mathrm{ng} / \mathrm{mL}$. $^{12}$ With a standard deviation (sigma) of $27 \mathrm{ng} / \mathrm{mL}$, a significance level $(2 \alpha)$ at 0.05 , and a power $(1-\beta)$ of $90 \%$, a total of 48 individuals were needed in each group.

\section{Results}

In total, 48 ET patients were included in the study and matched by age with 48 CAD patients previously studied by our research group. ${ }^{12}$-Table 1 shows the baseline characteristics of the two patient groups. A relatively low number of males were included in the ET group (42\%) compared with the CAD group (83\%). Furthermore, $8 \%$ of ET patients were current smokers compared with $27 \%$ of CAD patients.

\section{Serum TXB 2}

The primary outcome was the difference in delta values of $\mathrm{TXB}_{2}$ levels between the two patient groups, as shown in - Table 2. ET patients had significantly higher delta levels of $\mathrm{TXB}_{2}$ levels than CAD patients, demonstrating reduced suppression of $\mathrm{TXB}_{2}$. Median of differences in delta values of $\mathrm{TXB}_{2}$ levels between the two groups was $22.5 \mathrm{ng} / \mathrm{mL}$, $p<0.0001$.

We found significantly higher $\mathrm{TXB}_{2}$ levels 1 hour after aspirin intake in ET patients than in CAD patients (median: 6.4 [IQR: $3.1-14.4$ ] vs. median: 1.5 [IQR: $0.85-3.8$ ] ng/mL, $p<0.0001)$. In addition, ET patients had higher $\mathrm{TXB}_{2}$ levels 24 hours after aspirin intake than CAD patients (median: 32.3 [IQR: 14.2-64.0] vs. median: 5.1 [IQR: $3.8-9.6$ ] ng/mL, $p<0.0001$ ).

When dividing $\mathrm{TXB}_{2}$ values with platelet count to express $\mathrm{TXB}_{2}$ levels per unit of platelets, ET patients still had statistically significant higher levels when comparing delta values (median: 0.051 [IQR: 0.021-0.088] vs. median: 0.016 [IQR: 0.009-0.024], $p<0.0001$ ) and 24 hours levels (median: 0.069 [IQR: 0.039-0.107] vs. median: 0.024 [IQR: 0.016-0.046], $p<0.0001)$. $\mathrm{TXB}_{2}$ levels 1 hour after aspirin intake showed the same tendency but did not reach statistical significance (median: 0.012 [IQR: 0.007-0.024] vs. median: 0.007 [IQR: 0.004-0.017], $p<0.078$ ).

Table 1 Baseline characteristics of the study population with 48 patients with essential thrombocythemia (ET) and 48 patients with coronary artery disease (CAD)

\begin{tabular}{|c|c|c|c|}
\hline & ET patients & CAD patients & Reference interval \\
\hline \multicolumn{4}{|l|}{ Demographics } \\
\hline Age, y & $64.5(52.0-72.0)$ & $63.0(54.0-68.0)$ & \\
\hline Male sex, $n(\%)$ & $20(41.7)$ & $40(83.3)$ & \\
\hline Current smokers, $n(\%)$ & $4(8.3)$ & $13(27.1)$ & \\
\hline Body mass index, $\mathrm{kg} \mathrm{m}^{-2}$ & $24.2(21.4-26.8)$ & $27.7(25.1-28.7)$ & $18.5-25.0^{\mathrm{a}}$ \\
\hline Previously thromboembolic complications, $n$ (\%) & $5(10.4)$ & $48(100)$ & \\
\hline \multicolumn{4}{|l|}{ Mutation status } \\
\hline JAK2 mutation, $n(\%)$ & $32(66.7)$ & & \\
\hline CALR mutation, $n(\%)$ & $12(25.0)$ & & \\
\hline MPL mutation or triple negative, $n(\%)$ & $4(8.3)$ & & \\
\hline Time from diagnosis, y & 6.1 & & \\
\hline \multicolumn{4}{|l|}{ Biochemistry } \\
\hline Hemoglobin, mmol/L & $8.6(8.1-9.0)$ & $9.2(8.7-9,5)$ & $7.3-10.5^{b}$ \\
\hline Leucocyte count, $\times 10^{9} / \mathrm{L}$ & $6.0(4.9-9.0)$ & $6.3(5.2-7.8)$ & $3.5-10.0$ \\
\hline Platelet count, $\times 10^{9} / \mathrm{L}$ & $512.5(393.8-623.5)$ & $230.5(196.3-282.8)$ & $145-400^{\mathrm{b}}$ \\
\hline Mean platelet volume, $\mathrm{fL}$ & $9.8(9.1-10.5)$ & $10.8(10.3-11.5)$ & $6.5-12.0^{\mathrm{b}}$ \\
\hline Platelet large cell ratio, \% & $23.1(18.7-29.4)$ & $30.9(27.3-37.8)$ & $11.9-66.9$ \\
\hline Platelet distribution width, $\mathrm{fL}$ & $10.9(9.8-12.8)$ & $13.0(11.8-15.0)$ & $9.9-16.1^{b}$ \\
\hline $\begin{array}{l}\text { High immature platelet } \\
\text { fraction, \% }\end{array}$ & $1.2(0.7-1.5)$ & $0.7(0.5-1.0)$ & $0.1-2.7$ \\
\hline Creatinine, $\mu \mathrm{mol} / \mathrm{L}$ & $66(60.0-78.8)$ & $73.5(65.3-81.8)$ & $45-105^{b}$ \\
\hline
\end{tabular}

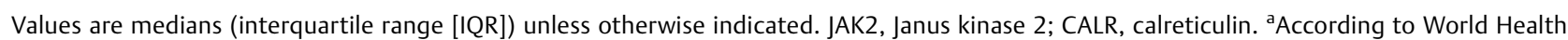

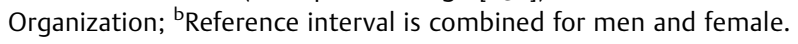


Table 2 Platelet function, reflecting the antiplatelet effect of aspirin, analyzed by platelet aggregation and serum thromboxane $B_{2}$ measurements ( $n=48$ in both patient groups)

\begin{tabular}{|c|c|c|c|c|c|c|c|}
\hline \multicolumn{3}{|l|}{ ET patients } & \multicolumn{3}{|c|}{ CAD patients } & \multirow[b]{2}{*}{ Comparison $^{\mathrm{a}}$} & \multirow[b]{2}{*}{$p$-Value ${ }^{\mathrm{b}}$} \\
\hline & & Difference & & & Difference & & \\
\hline $1 \mathrm{~h}$ & $24 \mathrm{~h}$ & (24 vs. $1 \mathrm{~h}$ ) & $1 \mathrm{~h}$ & $24 \mathrm{~h}$ & (24 vs. $1 \mathrm{~h})$ & ET vs. CAD & \\
\hline \multicolumn{8}{|c|}{ Thromboxane $B_{2} ; \mathrm{TXB}_{2},(\mathrm{ng} / \mathrm{mL})$} \\
\hline 6.4 & 32.3 & 26.2 & 1.5 & 5.1 & 3.7 & 22.5 & $<0.0001$ \\
\hline$(3.1-14.4)$ & $(14.2-64.0)$ & $(10.1-48.0)$ & $(0.8-3.6)$ & $(3.8-8.3)$ & $(2.6-6.0)$ & & \\
\hline \multicolumn{8}{|c|}{ Platelet aggregation; $\mathrm{AA},\left(\mathrm{AU}^{*} \mathrm{~min}\right)$} \\
\hline 455 & 786 & 251 & 434 & 522 & 119.5 & 131 & 0.0003 \\
\hline$(258-615)$ & $(474-934)$ & $(99-362)$ & $(266-595)$ & $(400-696)$ & $(52-192)$ & & \\
\hline
\end{tabular}

Abbreviations: AA, arachidonic acid (ASPItest); AU*min, aggregation units * min.; CAD, coronary artery disease; ET, essential thrombocythemia; TXB2, thromboxane B2.

Data are shown as median (interquartile range).

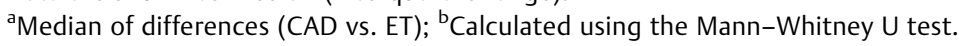

\section{Platelet Aggregation}

The differences in platelet aggregation from 1 hour after intake of aspirin to 24 hours after intake of aspirin were compared between the two patient groups. We demonstrated a significantly higher difference in platelet aggregation during 24 hours in ET patients than in CAD patients, as shown in -Table 2. Median of differences in platelet aggregation was $131 \mathrm{AU}^{*} \min , p=0.0003$.

We found no difference in platelet aggregation 1 hour after aspirin intake between the two patient-groups (median: 455 [IQR: 258-615] vs. median: 434 [IQR: 266-595] $\mathrm{ng} / \mathrm{mL}, p=0.68)$. ET patients had significantly higher platelet aggregation values 24 hours after aspirin intake than CAD patients (median: 785 [IQR: 474-934] vs. median: 522 [IQR: 400-696] ng/mL, $p=0.021$ ).

We attempted to correct platelet aggregation values for number of platelets. ET patients had significantly lower platelet aggregation 1 hour after aspirin intake (median: 0.88 [IQR: 0.53-1.21] vs. median: 1.74 [IQR: 1.29-2.44] ng/mL, $p<0.0001$ ) and 24 hours after aspirin intake than CAD patients (median: 1.33 [IQR: 0.91-1.84] vs. median: 2.27 [IQR: 1.82$2.78] \mathrm{ng} / \mathrm{mL}, p<0.0001$ ) when dividing platelet aggregation values with platelet count. No difference was found in platelet aggregation during 24 hours between ET patients and CAD patients when dividing platelet aggregation values with platelet count. During 24 hours, platelet aggregation and platelet count were significantly correlated in ET patients $(\mathrm{rho}=0.41$, $p=0.003$ ), but not in CAD patients (rho $=0.05, p=0.74$; -Fig. 1). At 24 hours following aspirin intake, platelet aggregation and platelet count were significantly correlated in ET patients (rho $=0.53, p<0.001$ ) and in CAD patients (rho $=0.48, p=0.001$; - Fig. 1). At 1 hour following aspirin intake, platelet aggregation and platelet count were not significantly correlated in ET patients ( $\mathrm{rho}=0.27, p=0.06$ ), but significantly correlated in CAD patients ( rho $=0.41, p=0.004$; - Fig. 1 ).

\section{Platelet Turnover}

As demonstrated in - Fig. 2, IPC was significantly higher in ET patients than in CAD patients (median: 18.8 [IQR: 11.6-29.4] vs. median: 5.7 [IQR: 4.2-7.3] x10 $10^{9} \mathrm{~L}, p<0.0001$ ). Furthermore, IPF was also significantly higher in ET patients than in CAD patients (median: 3.7 [IQR: 2.4-4.8] vs. median: 2.6 [IQR: $\left.1.8-3.3] \times 10^{9} / \mathrm{L}, p=0.0004\right)$.

As demonstrated in -Fig. 3, a statistically significant correlation between IPC and difference in platelet aggregation during 24 hours in ET patients was found (rho $=0.35$, $p=0.01)$. No other significant correlations were observed between IPC and differences in $\mathrm{TXB}_{2}$ levels or platelet aggregation during 24 hours in ET or CAD patients (-Fig. 3). No correlations between IPF and $\mathrm{TXB}_{2}$ levels or platelet aggregation (rho $=-0.19$ to $0.24, p>0.10$ ) were found in ET or CAD patients.

\section{Discussion}

We investigated the antiplatelet effect of aspirin in ET patients compared with stable CAD patients. Both patient groups have increased risk of thromboembolic complications and a reduced effect of once-daily aspirin monotherapy; however, the underlying mechanisms likely differ. Since ET is characterized as a platelet disorder, is it reasonable to assume that platelets may play a more important role in ET than in CAD for the risk of thromboembolic complications. The main finding of our study was that ET patients have significantly lower antiplatelet effect of aspirin during 24 hours than CAD patients. Furthermore, ET patients demonstrated higher platelet turnover, indicated by increased IPF and IPC compared with CAD patients.

We used two different methods to investigate the antiplatelet effect of aspirin: serum $\mathrm{TXB}_{2}$ measurements and platelet aggregation measured by impedance aggregometry. Both methods showed significantly lower antiplatelet effect of aspirin during 24 hours in ET patients compared with CAD patients. Determination of $\mathrm{TXB}_{2}$ levels is considered the most pharmacologically specific way to evaluate the antiplatelet effect of aspirin, because the contribution to its synthesis from other blood cells is minimal. ${ }^{24}$ Additionally, dividing $\mathrm{TXB}_{2}$ measurements with platelet count had overall no 
A. ET patients

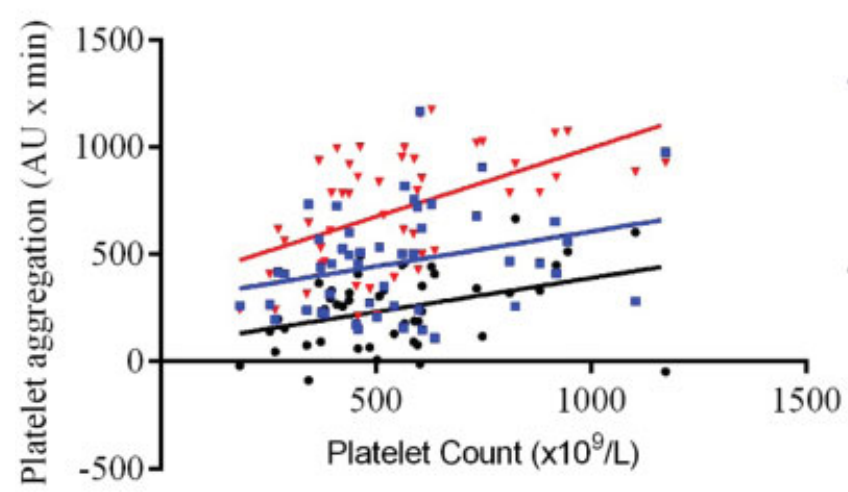

- 24 hours after aspirin intake

$$
\text { rho }=0.53
$$$$
p<0.001
$$

$\rightarrow 1$ hour after aspirin intake

$$
\begin{aligned}
& \text { rho }=0.27 \\
& p=0.06
\end{aligned}
$$

Difference between 24 hours

and 1 hour

$$
\text { rho }=0.41
$$

$$
p=0.003
$$

\section{A. CAD patients}

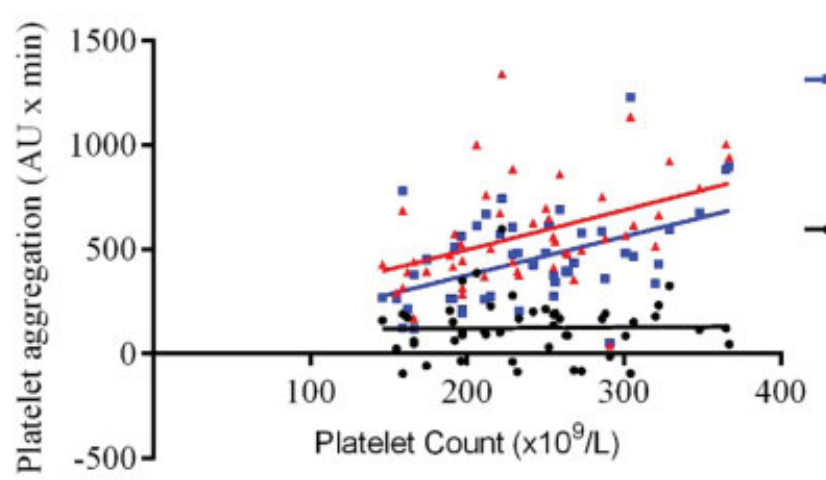

- 24 hours after aspirin intake

$$
\begin{aligned}
& \text { rho }=0.48 \\
& p=0.001
\end{aligned}
$$

1 hour after aspirin intake

$$
\begin{aligned}
& \text { rho }=0.41 \\
& p=0.004
\end{aligned}
$$

$\rightarrow$ Difference between 24 hours and 1 hour

$$
\begin{aligned}
& \text { rho }=0.05 \\
& p=0.74
\end{aligned}
$$

Fig. 1 Correlation between platelet aggregation and platelet count: (1) 24 hours after aspirin intake, (2) 1 hour after aspirin intake, and ( 3 ) difference between 24 and 1 hour in A: 48 patients with essential thrombocythemia (ET) and B: 48 patients with coronary artery disease (CAD).

influence on the results. Hence, $\mathrm{TXB}_{2}$ generation is inhibited to a lower extent throughout the day, indicating a lower antiplatelet response to aspirin treatment in ET patients than in CAD patients. A previous study demonstrated that increased platelet count contributed to increased residual TXB2 level prior to aspirin treatment, but not after aspirin antiplatelet treatment. ${ }^{25}$ Platelet aggregation measured by impedance aggregometry is known to be positively associated with platelet count. ${ }^{26,27}$ Indeed, when platelet aggregation results were divided by platelet count, the difference in antiplatelet effect of aspirin during 24 hours was no longer present indicating that the significantly higher aggregation found could, at least in part, be explained by the higher platelet count in ET patients. However, simply dividing platelet aggregation with platelet count is an imperfect way of adjusting for high platelet count, although studies found a linear relationship between platelet count up to $700 \times 10^{9} / \mathrm{L}$ and platelet aggregation. ${ }^{26-29}$

Our finding of reduced effect of aspirin in ET patients is consistent with previous studies showing that monotherapy with low-dose aspirin results in insufficient platelet inhibition during the usual 24-hour dosing interval in ET patients. ${ }^{3,23}$ This might be partly explained by an increased platelet count, as studies have shown that the effect of aspirin depends on the platelet count. ${ }^{30,31}$ Furthermore, the type of aspirin may also be of importance, as plain aspirin may provide more efficient platelet inhibition than enteric-coated aspirin in patients with ET. ${ }^{32}$ Aspirin exerts its effect by irreversible acetylation of the platelet COX resulting in inhibition of $\mathrm{TXA}_{2}$ biosynthesis lasting for an entire platelet life span. ${ }^{33}$ Hence, the irreversible inhibition of aspirin compensates for the short half-life., ${ }^{3,34}$ Under normal thrombopoietic conditions without increased platelet turnover, once daily aspirin provides sufficient platelet inhibition..$^{34}$ In addition, the irreversible inactivation of COX by aspirin also exerts its effect on the progenitor cells in the bone marrow, thus newly formed platelets are mainly nonfunctioning throughout the day. ${ }^{35-37}$ However, patients with increased platelet turnover have a considerable release of platelets unaffected by aspirin into the bloodstream, ${ }^{11}$ thus counteracting the irreversible COX inhibition. ${ }^{11}$ In ET patients, studies have demonstrated an accelerated renewal of the drug target as a possible explanation for the reduced antiplatelet effect of aspirin. ${ }^{3,5,38} \mathrm{~A}$ doubling of the aspirin dose given once daily may increase platelet inhibition immediately after administration, but still fails to provide 


\section{Immature platelet count}

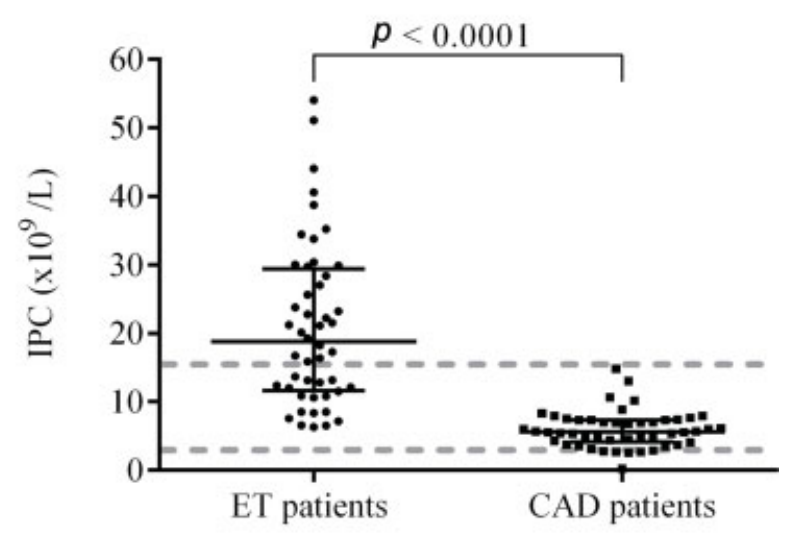

Immature platelet fraction

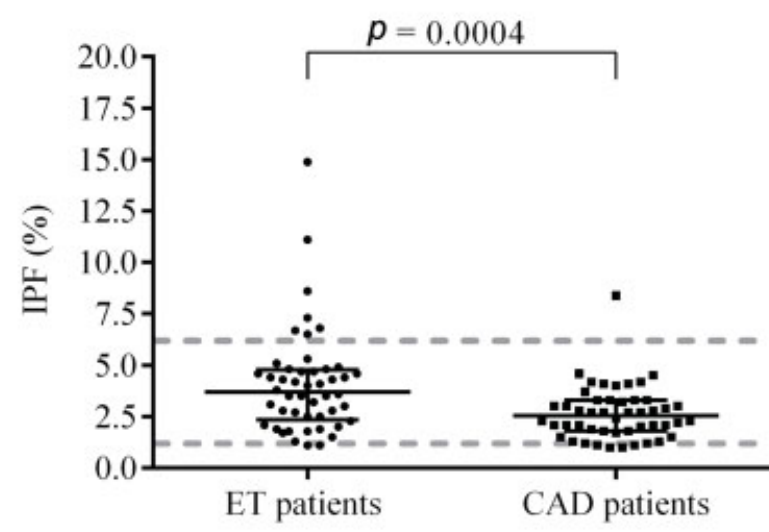

Grey dotted lines indicate reference intervals.

Fig. 2 Comparison of immature platelet count (IPC) and immature platelet fraction (IPF) in 48 patients with essential thrombocythemia (ET) and 48 patients with coronary artery disease (CAD).

sufficient 24-hour platelet inhibition. ${ }^{3,39}$ Therefore, to obtain a more consistent platelet inhibition throughout the day, the dose interval should be decreased. ${ }^{16,23}$ Accordingly, other studies on ET patients have shown that a twice-daily dosing of aspirin provides a more consistent platelet inhibition during 24 hours., ${ }^{3,16}$ Furthermore, data from a large randomized controlled trial ${ }^{16}$ indicate that twice-daily dosing of aspirin reduces the residual $\mathrm{TXB}_{2}$ level with around $90 \%,{ }^{40}$ whereas once-daily dosing of aspirin was insufficient in up to $80 \%$ of ET patients. ${ }^{5}$ Notably, even a very low-dose of aspirin given twice daily $(37.5 \mathrm{mg})$ causes consistent platelet inhibition during 24 hours in ET patients. ${ }^{23}$ In a new guideline, twice-daily aspirin treatment is now recommended for ET patients with arterial thrombosis if they are older than 65 years, have a Janus kinase 2 mutation, or have cardiovascular risk factors, as these factors have shown to be the major risk factors for thrombosis. ${ }^{1,41}$ However, the ability of aspirin to prevent thromboembolic events in ET patients is still unclear and might be limited to only some subgroups of ET patients, ${ }^{42-45}$ as low-dose aspirin in patients with very high platelet count may result in paradoxical occurrence of thrombosis and bleeding. ${ }^{46-48}$ ET patients may also benefit from dual antiplatelet therapy with aspirin plus an adenosine diphosphate-inhibitor, ${ }^{49,50}$ although only few studies have investigated this hypothesis.

The prothrombotic properties of immature platelets per se may also contribute to the reduced effect of aspirin. ${ }^{11,19,20}$ Additionally, several studies have reported an association between increased levels of immature platelets and a high residual platelet activity evaluated by $\mathrm{TXB}_{2}$ measurements or platelet aggregation ${ }^{18,51,52}$ In this study, IPC showed a statistically significant, though only moderately strong, positive correlation with platelet aggregation measured by impedance aggregometry in ET patients. No other significant correlations were observed between immature platelet markers and platelet function test in either of the patient groups. This is in accordance with previous studies, indicating that the absolute number rather than the relative fraction of immature platelets has a stronger correlation with platelet aggregation and $\mathrm{TXB}_{2}$ levels. ${ }^{3,53,54}$ In addition, studies have demonstrated that especially ET patients with a history of thromboembolic complications have an increased platelet turnover compared with ET patients without thromboembolic complications..$^{55,56}$ Our study was not sufficiently powered to address this. Yet, it is still plausible that both the prothrombotic properties of immature platelets per se and an increased platelet turnover leading to the release of platelets unaffected by aspirin into the blood stream are concurrent mechanisms explaining our findings.

This study is the first study comparing strictly the WHOdefined ET patients with CAD patients to examine the importance of platelets to the antiplatelet effect of aspirin. We used two different methods to investigate the antiplatelet effect of aspirin, that is, serum $\mathrm{TXB}_{2}$ measurements and platelet aggregation. However, some limitations have to be considered. It should be acknowledged that CAD patients were originally included in a previous study. ${ }^{12}$ As the two cohorts were only matched by age, they are likely to differ in other aspects. Different gender distribution was observed in the two groups, which may have influenced the results, as some studies have indicated that aspirin-treated women may more often have increased on-treatment platelet reactivity. ${ }^{57,58}$ In addition, as smoking increases platelet aggregation, the differences in platelet aggregation observed between ET patients and CAD patients may be slightly underestimated as there were more smokers among CAD patients than ET patients. ${ }^{59}$ We studied platelet aggregation in vitro, whereas the present study was not powered to assess clinical events. As we only included patients treated with aspirin monotherapy, our results may not be extrapolated to patients receiving other antithrombotic regimens.

\section{Conclusion}

ET patients have reduced 24-hour antiplatelet effect of oncedaily aspirin and an increased platelet turnover compared with CAD patients. Hence, platelets are possibly more 
A. ET patients

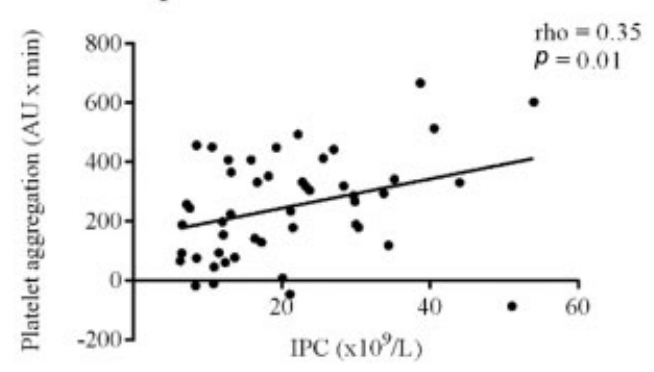

B. CAD patients

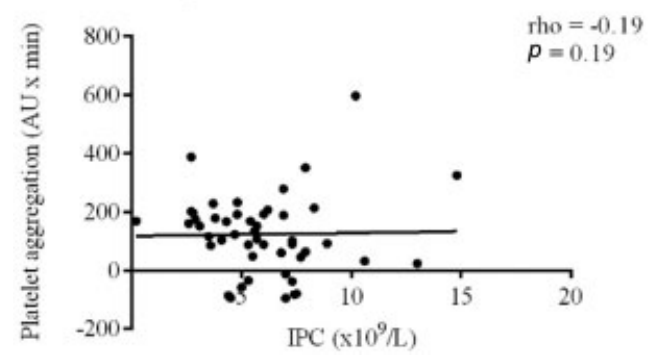

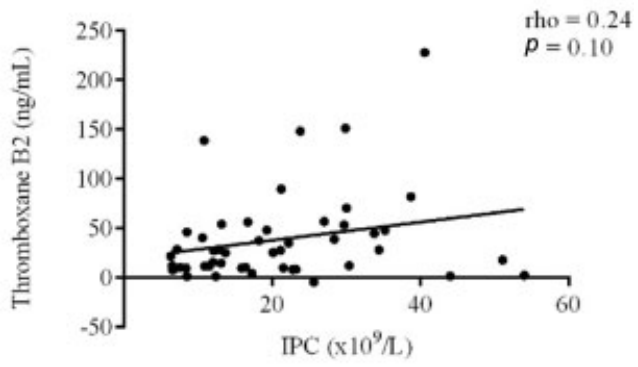

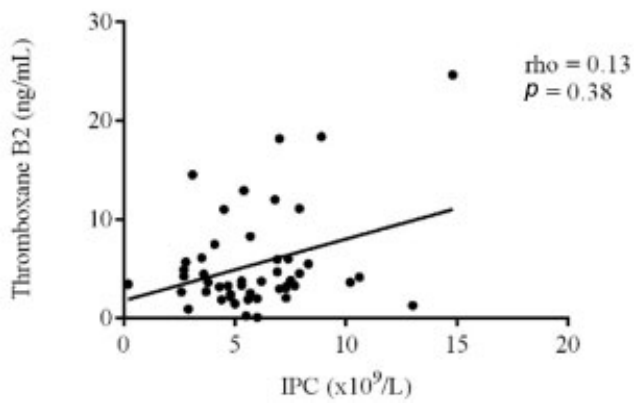

Fig. 3 Correlation between immature platelet count (IPC) and (1) difference in platelet aggregation between 24 and 1 hour and (2) difference in thromboxane $B_{2}$ levels between 24 and 1 hour in A: 48 patients with essential thrombocythemia (ET) and B: 48 patients with coronary artery disease (CAD).

important for the risk of thromboembolic complications in ET than in CAD. These findings strengthen the rationale for exploring novel antiplatelet drugs or aspirin dosing regimens ${ }^{40}$ to overcome the reduced 24-hour antiplatelet effect of aspirin and reduce the risk of cardiovascular events.

\section{Competing Interests}

None related to this manuscript. The authors report the following general conflicts:

ELG has received speaker honoraria or consultancy fees from AstraZeneca, Bayer, Boehringer Ingelheim, BristolMyers Squibb, Pfizer, MSD, Mundipharma, Portola Pharmaceuticals, and Roche. He is an investigator in the SATELLITE, FLAVOUR, and ETESIAN studies (AstraZeneca) and has received unrestricted research grants from Boehringer Ingelheim. SDK has received lecture fees from Aspen and AstraZeneca. AMH has received speaker's fees from CSL Behring, Bayer, Boehringer-Ingelheim, Bristol-Myers Squibb, and Leo Pharma and unrestricted research support from Octapharma, and CSL Behring. OBP and HBO have no conflicts to declare.

\section{Acknowledgments}

The authors would like to acknowledge MD, PhD, Morten Würtz for collecting the data on CAD patients. Furthermore, the authors would like to acknowledge laboratory technicians Mai Stenulm Veirup and Vivi Bo Mogensen for their assistance in the laboratory.
The study was financially supported by the Oda and Hans Svenningsens Foundation. SDK was supported by a grant from the Novo-Nordic Foundation [NNF140C0008817]. The funding sources had no influence on the study.

All authors designed the research study; OBP performed the research; all authors analyzed the data and contributed to writing the paper.

\section{References}

1 Tefferi A, Barbui T. Polycythemia vera and essential thrombocythemia: 2019 update on diagnosis, risk-stratification and management. Am J Hematol 2019;94(01):133-143

2 Baigent C, Blackwell L, Collins R, et al; Antithrombotic Trialists' (ATT) Collaboration. Aspirin in the primary and secondary prevention of vascular disease: collaborative meta-analysis of individual participant data from randomised trials. Lancet 2009;373 (9678):1849-1860

3 Pascale S, Petrucci G, Dragani A, et al. Aspirin-insensitive thromboxane biosynthesis in essential thrombocythemia is explained by accelerated renewal of the drug target. Blood 2012;119(15): 3595-3603

4 Carobbio A, Thiele J, Passamonti F, et al. Risk factors for arterial and venous thrombosis in WHO-defined essential thrombocythemia: an international study of 891 patients. Blood 2011;117 (22):5857-5859

5 Dragani A, Pascale S, Recchiuti A, et al. The contribution of cyclooxygenase- 1 and -2 to persistent thromboxane biosynthesis in aspirin-treated essential thrombocythemia: implications for antiplatelet therapy. Blood 2010;115(05):1054-1061

6 Krasopoulos G, Brister SJ, Beattie WS, Buchanan MR. Aspirin "resistance" and risk of cardiovascular morbidity: systematic review and meta-analysis. BMJ 2008;336(7637):195-198 
7 Snoep JD, Hovens MM, Eikenboom JC, van der Bom JG, Huisman MV. Association of laboratory-defined aspirin resistance with a higher risk of recurrent cardiovascular events: a systematic review and meta-analysis. Arch Intern Med 2007;167(15): 1593-1599

8 Dillinger JG, Sideris G, Henry P, Bal dit Sollier C, Ronez E, Drouet L. Twice daily aspirin to improve biological aspirin efficacy in patients with essential thrombocythemia. Thromb Res 2012; 129(01):91-94

9 Schafer AI. Molecular basis of the diagnosis and treatment of polycythemia vera and essential thrombocythemia. Blood 2006; 107(11):4214-4222

10 Budde U, Dent JA, Berkowitz SD, Ruggeri ZM, Zimmerman TS. Subunit composition of plasma von Willebrand factor in patients with the myeloproliferative syndrome. Blood 1986;68(06): 1213-1217

11 Freynhofer MK, Gruber SC, Grove EL, Weiss TW, Wojta J, Huber K. Antiplatelet drugs in patients with enhanced platelet turnover: biomarkers versus platelet function testing. Thromb Haemost 2015;114(03):459-468

12 Würtz M, Hvas AM, Jensen LO, et al. 24-hour antiplatelet effect of aspirin in patients with previous definite stent thrombosis. Int J Cardiol 2014;175(02):274-279

13 Lordkipanidzé M, Pharand C, Schampaert E, Palisaitis DA, Diodati $\mathrm{JG}$. Heterogeneity in platelet cyclooxygenase inhibition by aspirin in coronary artery disease. Int J Cardiol 2011;150(01):39-44

14 Rocca B, Santilli F, Pitocco D, et al. The recovery of platelet cyclooxygenase activity explains interindividual variability in responsiveness to low-dose aspirin in patients with and without diabetes. J Thromb Haemost 2012;10(07):1220-1230

15 Tefferi A, Pardanani A. Essential thrombocythemia. N Engl J Med 2019;381(22):2135-2144

16 De Stefano V, Rocca B, Tosetto A, et al. The Aspirin Regimens in Essential Thrombocythemia (ARES) phase II randomized trial design: implementation of the serum thromboxane $B_{2}$ assay as an evaluation tool of different aspirin dosing regimens in the clinical setting. Blood Cancer J 2018;8(06):49

17 McBane RD II, Gonzalez C, Hodge DO, Wysokinski WE. Propensity for young reticulated platelet recruitment into arterial thrombi. J Thromb Thrombolysis 2014;37(02):148-154

18 Guthikonda S, Alviar CL, Vaduganathan M, et al. Role of reticulated platelets and platelet size heterogeneity on platelet activity after dual antiplatelet therapy with aspirin and clopidogrel in patients with stable coronary artery disease. J Am Coll Cardiol 2008;52 (09):743-749

19 Cesari F, Marcucci R, Caporale R, et al. Relationship between high platelet turnover and platelet function in high-risk patients with coronary artery disease on dual antiplatelet therapy. Thromb Haemost 2008;99(05):930-935

20 Rocca B, Secchiero P, Ciabattoni G, et al. Cyclooxygenase-2 expression is induced during human megakaryopoiesis and characterizes newly formed platelets. Proc Natl Acad Sci U S A 2002;99(11): 7634-7639

21 Swerdlow SHCE, Harris NL, Jaffe ES, Pileri SA, Stein H, Thiele J. WHO Classification of Tumours of Haematopoietic and Lymphoid Tissues. Vol 2. Revised 4th Edition ed. Lyon, France: International Agency for Research on Cancer (IARC); 2017

22 Tóth O, Calatzis A, Penz S, Losonczy H, Siess W. Multiple electrode aggregometry: a new device to measure platelet aggregation in whole blood. Thromb Haemost 2006;96(06):781-788

23 Larsen ML, Pedersen OH, Hvas AM, et al. Once- versus twice-daily aspirin treatment in patients with essential thrombocytosis. Platelets 2019;30(03):322-328

24 Cattaneo M. Laboratory detection of 'aspirin resistance': what test should we use (if any)? Eur Heart J 2007;28(14):1673-1675

25 Frelinger AL, Li Y, Linden MD, et al. Aspirin 'resistance': role of preexistent platelet reactivity and correlation between tests. J Thromb Haemost 2008;6(12):2035-2044
26 Würtz M, Hvas AM, Kristensen SD, Grove EL. Platelet aggregation is dependent on platelet count in patients with coronary artery disease. Thromb Res 2012;129(01):56-61

27 Pedersen OH, Larsen ML, Grove EL, et al. Platelet characteristics in patients with essential thrombocytosis. Cytometry B Clin Cytom 2018;94(06):918-927

28 Hanke AA, Roberg K, Monaca E, et al. Impact of platelet count on results obtained from multiple electrode platelet aggregometry (Multiplate). Eur J Med Res 2010;15(05):214-219

29 Femia EA, Scavone M, Lecchi A, Cattaneo M. Effect of platelet count on platelet aggregation measured with impedance aggregometry (Multiplate ${ }^{\mathrm{TM}}$ analyzer) and with light transmission aggregometry. J Thromb Haemost 2013;11(12):2193-2196

30 Gremmel T, Gisslinger B, Gisslinger H, Panzer S. Response to aspirin therapy in patients with myeloproliferative neoplasms depends on the platelet count. Transl Res 2018;200:35-42

31 Petrides PE, Siegel F. Thrombotic complications in essential thrombocythemia (ET): clinical facts and biochemical riddles. Blood Cells Mol Dis 2006;36(03):379-384

32 Scavone M, Rizzo J, Femia EA, et al. Patients with essential thrombocythemia may be poor responders to enteric-coated aspirin, but not to plain aspirin. Thromb Haemost 2020;120 (10):1442-1453

33 Patrono C, García Rodríguez LA, Landolfi R, Baigent C. Low-dose aspirin for the prevention of atherothrombosis. $\mathrm{N}$ Engl J Med 2005;353(22):2373-2383

34 Schrör K. Aspirin and platelets: the antiplatelet action of aspirin and its role in thrombosis treatment and prophylaxis. Semin Thromb Hemost 1997;23(04):349-356

35 Giaretta A, Rocca B, Di Camillo B, Toffolo GM, Patrono C. In silico modeling of the antiplatelet pharmacodynamics of low-dose aspirin in health and disease. Clin Pharmacol Ther 2017;102 (05):823-831

36 Burch JW, Stanford N, Majerus PW. Inhibition of platelet prostaglandin synthetase by oral aspirin. J Clin Invest 1978;61(02): 314-319

37 Santilli F, Rocca B, De Cristofaro R, et al. Platelet cyclooxygenase inhibition by low-dose aspirin is not reflected consistently by platelet function assays: implications for aspirin "resistance". J Am Coll Cardiol 2009;53(08):667-677

38 Patrono C, Rocca B, De Stefano V. Platelet activation and inhibition in polycythemia vera and essential thrombocythemia. Blood 2013;121(10):1701-1711

39 Perneby C, Wallén NH, Rooney C, Fitzgerald D, Hjemdahl P. Doseand time-dependent antiplatelet effects of aspirin. Thromb Haemost 2006;95(04):652-658

40 Rocca B, Tosetto A, Betti S, et al. A randomized double-blind trial of 3 aspirin regimens to optimize antiplatelet therapy in essential thrombocythemia. Blood 2020;136(02):171-182

41 Landolfi R, Di Gennaro L, Novarese L, Patrono C. Aspirin for the control of platelet activation and prevention of thrombosis in essential thrombocythemia and polycythemia vera: current insights and rationale for future studies. Semin Thromb Hemost 2006;32(03):251-259

42 Chu DK, Hillis CM, Leong DP, Anand SS, Siegal DM. Benefits and risks of antithrombotic therapy in essential thrombocythemia: a systematic review. Ann Intern Med 2017;167(03):170-180

43 Alvarez-Larrán A, Cervantes F, Pereira A, et al. Observation versus antiplatelet therapy as primary prophylaxis for thrombosis in low-risk essential thrombocythemia. Blood 2010;116(08): 1205-1210, quiz 1387

44 Alvarez-Larrán A, Pereira A, Guglielmelli P, et al. Antiplatelet therapy versus observation in low-risk essential thrombocythemia with a CALR mutation. Haematologica 2016;101(08): 926-931

45 De Stefano V, Rossi E, Carobbio A, et al. Hydroxyurea prevents arterial and late venous thrombotic recurrences in patients with myeloproliferative neoplasms but fails in the splanchnic venous 
district. Pooled analysis of 1500 cases. Blood Cancer J 2018;8(11): 112

46 Michiels JJ. Myeloproliferative and thrombotic burden and treatment outcome of thrombocythemia and polycythemia patients. World J Crit Care Med 2015;4(03):230-239

47 van Genderen PJ, Michiels JJ, van der Poel-van de Luytgaarde SC, van Vliet HH. Acquired von Willebrand disease as a cause of recurrent mucocutaneous bleeding in primary thrombocythemia: relationship with platelet count. Ann Hematol 1994;69(02): 81-84

48 Michiels JJ, Berneman ZN, Schroyens W, Van Vliet HH. Pathophysiology and treatment of platelet-mediated microvascular disturbances, major thrombosis and bleeding complications in essential thrombocythaemia and polycythaemia vera. Platelets 2004;15 (02):67-84

49 Nurden P, Bihour C, Smith M, Raymond JM, Nurden AT. Platelet activation and thrombosis: studies in a patient with essential thrombocythemia. Am J Hematol 1996;51(01):79-84

50 Panova-Noeva M, Marchetti M, Russo L, et al. ADP-induced platelet aggregation and thrombin generation are increased in essential thrombocythemia and polycythemia vera. Thromb Res 2013;132(01):88-93

51 Würtz M, Grove EL, Wulff LN, et al. Patients with previous definite stent thrombosis have a reduced antiplatelet effect of aspirin and a larger fraction of immature platelets. JACC Cardiovasc Interv 2010;3(08):828-835
52 Guthikonda S, Lev EI, Patel R, et al. Reticulated platelets and uninhibited COX-1 and COX-2 decrease the antiplatelet effects of aspirin. J Thromb Haemost 2007;5(03):490-496

53 Stratz C, Bömicke T, Younas I, et al. Comparison of immature platelet count to established predictors of platelet reactivity during thienopyridine therapy. J Am Coll Cardiol 2016;68(03): 286-293

54 Grove EL, Würtz M, Hvas AM, Kristensen SD. Increased platelet turnover in patients with previous definite stent thrombosis. J Thromb Haemost 2011;9(07):1418-1419

55 Arellano-Rodrigo E, Alvarez-Larrán A, Reverter JC, et al. Platelet turnover, coagulation factors, and soluble markers of platelet and endothelial activation in essential thrombocythemia: relationship with thrombosis occurrence and JAK2 V617F allele burden. Am J Hematol 2009;84(02):102-108

56 Rinder HM, Schuster JE, Rinder CS, Wang C, Schweidler HJ, Smith BR. Correlation of thrombosis with increased platelet turnover in thrombocytosis. Blood 1998;91(04):1288-1294

57 Marcucci R, Cioni G, Giusti B, et al. Gender and anti-thrombotic therapy: from biology to clinical implications. J Cardiovasc Transl Res 2014;7(01):72-81

58 Rubak P, Villadsen K, Hvas AM. Reference intervals for platelet aggregation assessed by multiple electrode platelet aggregometry. Thromb Res 2012;130(03):420-423

59 Glynn MF, Mustard JF, Buchanan MR, Murphy EA. Cigarette smoking and platelet aggregation. Can Med Assoc J 1966;95(11):549-553 